Review Article

\title{
Combined Raman Spectroscopy and Digital Holographic Microscopy for Sperm Cell Quality Analysis
}

\author{
A. De Angelis, ${ }^{1}$ S. Managò, ${ }^{1}$ M. A. Ferrara, ${ }^{2}$ M. Napolitano, ${ }^{1}$ G. Coppola, ${ }^{2}$ and A. C. De Luca ${ }^{1}$ \\ ${ }^{1}$ Institute of Protein Biochemistry, National Research Council, Via P. Castellino 111, 80131 Naples, Italy \\ ${ }^{2}$ Institute for Microelectronics and Microsystems, National Research Council, Via P. Castellino 111, 80131 Naples, Italy \\ Correspondence should be addressed to A. C. De Luca; a.deluca@ibp.cnr.it
}

Received 20 September 2016; Revised 5 December 2016; Accepted 14 December 2016; Published 10 April 2017

Academic Editor: Christoph Krafft

Copyright (C) 2017 A. De Angelis et al. This is an open access article distributed under the Creative Commons Attribution License, which permits unrestricted use, distribution, and reproduction in any medium, provided the original work is properly cited.

\begin{abstract}
The diagnosis of male infertility is vastly complex. To date, morphology, motility, and concentration have been used as key parameters to establish the sperm normality and achieve pregnancy both in natural and in assisted fecundation. However, spermatozoa from infertile men could present a variety of alterations, such as DNA fragmentation, alterations of chromatin structure, and aneuploidy, which have been demonstrated to decrease reproductive capacity of men. Therefore, the ability to see detailed relationships between morphology and physiology in selected spermatozoa with submicrometric resolution in a nondestructive and noninvasive way and within a functional correlated context could be extremely important for the intracytoplasmic sperm injection procedure. In this review, we describe label-free optical spectroscopy and imaging techniques, based on the combination of Raman spectroscopy/imaging with holographic imaging, which are able to noninvasively measure the (bio)chemistry and morphology of sperm cells. We discuss the benefits and limitation of the proposed photonic techniques, with particular emphasis on applications in detection/characterization of sperm cell morphological defects and photodamage, and the identification/sorting of $\mathrm{X}$ - and Y-bearing bovine spermatozoa.
\end{abstract}

\section{Introduction}

Sperm cell analysis is an imperfect tool but remains the cornerstone of the investigation of male infertility [1]. The sperm selection is one of the most crucial aspects of the assisted reproductive techniques and an effective procedure for selecting normal sperm is greatly needed [2]. Routine semen analysis is based on the analysis of sperm cell morphology, motility, and concentration [3-8]. Although this assay reveals useful information for the initial evaluation of the infertile male, it is not a test of fertility. The standard selection protocols are not discriminatory with respect to the identification of spermatozoa with normal haploid chromosome or intact chromatin or DNA [9-11]. Sperm DNA integrity is assessed by destructive methods such as terminal deoxynucleotidyl transferase dUTP nick end labeling (TUNEL) assay [12], comet assay [13], sperm chromatin dispersion (SCD) test [14], or sperm chromatin structure assay (SCSA) [15], which are assays for the detection of damaged DNA or altered protein in sperm nuclei by using specific stains. Indeed, current sperm physiology tests are of limited clinical utility as they render the sample unusable [12-17].

Recently, spectroscopy has emerged as one of the major tools for biomedical applications and has made significant progress in the field of clinical evaluation. Research has been carried out on a number of human cells using spectroscopic techniques, including Raman spectroscopy. This vibrational spectroscopic technique does not require sample labelling, getting biochemical information directly from the inelastic light scattering induced by its molecular vibrations, and only small amounts of material (micrograms to nanograms) with a minimum sample preparation are required (water or culture medium can be used as solvent) [18-28]. This makes Raman spectroscopy a relatively simple, noninvasive, and nondestructive technique providing molecular-level information, allowing investigation of functional groups, bonding types, and molecular conformations [29-31]. Interestingly, due to the cited characteristics, it has been successfully employed for the study of several living/fixed 
cells [20-23, 30-33], including sperm cells [6-8, 25-28]. Huser et al., in a recent paper, studied membranous human sperm cells by Raman spectroscopy and correlated the nuclear shape (normal versus abnormal) with protein content and DNA packaging [27]. The Raman approach has been used to demonstrate that spectra could identify UVinduced [28] or oxidative nDNA damage [34], localize and map DNA damage [28], assess the mitochondrial status in human spermatozoa [25], and separate sperm cells that are bound to the human zona pellucida from unbound sperm cells [35]. In 2015, Edengeiser et al. showed that Raman spectroscopy allows to chemically assess single, living human spermatozoa in near-physiological conditions [26].

Additionally, Raman spectroscopy can be easily combined with complementary optical approaches, such as holographic microscopy $[7,8,36]$. Based on the refractive index difference between the cell and the surrounding medium, holographic microscopy analyses the phase of the light transmitted by the sample and allows 3D quantitative sample image reconstruction [37-42]. Compared with the established morphological approaches used in biology such as fluorescence microscopy or more sophisticated techniques for instance atomic force microscopy (AFM), holographic imaging has three potential advantages: (i) The entire volume information can be acquired in one shot, avoiding mechanical movements $[4,37,38,43]$. (ii) The reconstructed images can be exploited for quantitative microtopology (such as volume measurements) [37, 38, 44, 45]. (iii) The sample can be analysed in a physiological state $[39,40]$. Therefore, holographic microscopy enables the high contrast characterization of live specimen [38-40, 44, 46, 47].

Due to the holographic microscopy ability for quantitatively monitoring cell structure and dynamics, it has been used to address specific questions in the field of andrology research. Indeed, several configurations [44, 45, 48-50] have been implemented to track the principal features of sperm morphology and navigation in a 3D chemical landscape enabling accurate analysis of cell parameters such as $3 \mathrm{D}$ tomography, biovolume, curvilinear velocity, and straightline velocity.

The combination of both holographic microscopy and Raman measurements could further improve the sperm cell analysis monitoring simultaneously morphological and physiological parameters. A combined approach based on sequential measurements with the same laser probe has been proposed by Kang et al. [51] for the investigation of blood disorders. By using two separate laser probes, Pavillon et al. demonstrated for the first time the possibility to simultaneously measure the morphological live cell (HeLa cell) characteristics in real time during the Raman acquisition [36]. Thus, this combined imaging approach could be the perfect candidate for noninvasively and nondestructively selecting single, live spermatozoa for the intracytoplasmic sperm injection procedure.

It is the purpose of this review to highlight the analytical potential and the achieved results of the combined Raman spectroscopy/imaging approach and holographic microscopy for the morphological and physiological characterization of single selected sperm cells. The following topics will be discussed:
(1) Correlative Morphological and Physiological Characterization of Sperm Cell Defects. Firstly, we discuss the ability to correlate the detailed morphological information provided by the holographic images with the biochemical analysis provided by the Raman imaging approach.

(2) Raman Spectroscopy and Holographic Microscopy for Sperm Cell Sex Sorting. Secondly, we focus on recent achievements in the identification of X- and Ychromosome-bearing sperm cells by using the Raman/holography approaches.

(3) Simultaneous Anatomical and Biochemical Analysis of Sperm Cell Photodamage. Finally, we study the photodamage induced by visible irradiation in order to demonstrate the completeness of our approach in simultaneously detected morphological and biochemical photo-oxidative damages on the sperm cells.

In a concluding section, we will present our expectations regarding the future importance of the proposed combined photonic technique as an efficient method for healthy and fertile living sperm cell selection, without injuring the sample, improving assisted reproduction technique outcomes and increasing the pregnancy rate.

\section{Materials and Methods}

2.1. Raman Spectroscopy/Imaging. The Raman spectroscopy is a spectroscopic technique based on the principle of the Raman scattering. When the laser light interacts with the sample object, most photons are scattered at the same optical frequency (energy) of the incident one (Rayleigh scattering), while a small fraction (approximately 1/1000000), interacting with the molecular vibrations, can be scattered at different frequencies (inelastic scattering). The process leading to the inelastic scattering is known as the Raman effect, and the shift in energy between incident and scattered photons provides information on the vibrational modes in the analyte:

$$
\bar{v}=\frac{1}{\lambda_{\text {incident }}}-\frac{1}{\lambda_{\text {scattered }}} .
$$

By collecting the photons scattered at different frequencies, it is possible to reconstruct a Raman spectrum, that is, a plot of scattered light versus energy shift, which is a "fingerprint" of the sample allowing the molecule identifications without the use of labels or dyes. Thus, Raman spectroscopy offers several advantages for microscopic analysis of biological samples [21, 23-25, 27, 30, 32, 33, 35, 52].

By raster scanning the sample through the laser probe and acquiring thousands of Raman spectra from all over the field of view, it is possible to reconstruct a Raman image or a chemical map of the sample. Therefore, the spectra can then be used to generate images showing the location and amount of different components (false colour images) $[7,8,21,30,35,52,53]$.

2.2. Holographic Microscopy. Holographic microscopy is a technique based on the classic holographic principle. 


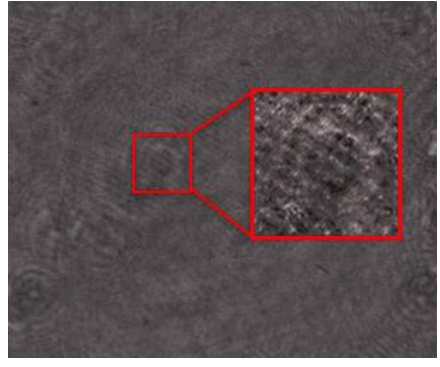

(a)

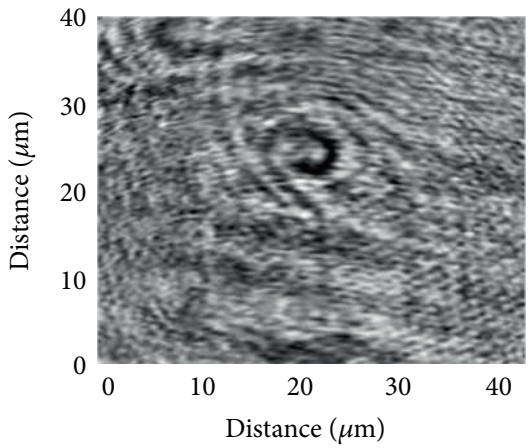

(c)

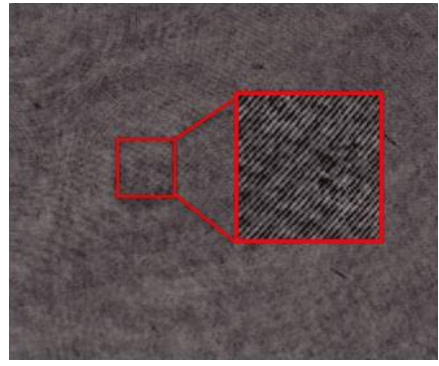

(b)

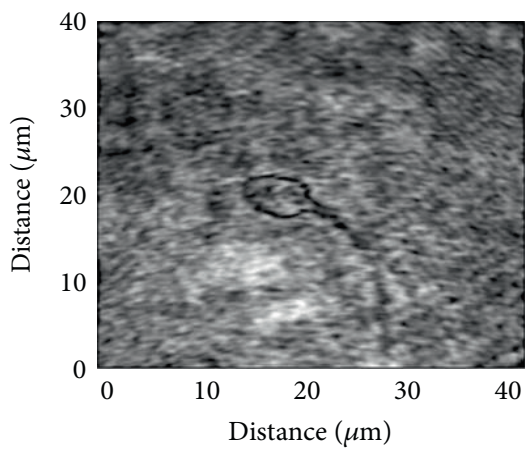

(d)

FIGURE 1: (a) Hologram of the sperm cell acquired out of focus. (b) Reference hologram acquired in the sperm cell proximity region. (c) Reconstructed amplitude map at the plane of acquisition. (d) Reconstructed amplitude map of the sperm cell region at the focus plane [8].

Basically, an image sensor (CCD or CMOS) acquires the hologram; that is, the interference pattern formed when a reference beam of fixed wavelength (coherent monochromatic light source) encounters light of the same fixed wavelength arriving from an object (the object beam). The scattered light by the sample object is

$$
O(x, y)=|O(x, y)| e^{i \varphi(x, y)},
$$

where $|O|$ and $\varphi$ are the wave field amplitude and phase in the hologram plane $(x, y)$, respectively. In a transmission mode, the phase shift is related to the optical path difference (OPD) by

$$
\Delta \varphi(x, y)=\frac{2 \pi}{\lambda} \mathrm{OPD}\left(n_{\text {object }}, n_{\text {medium }}, d_{\text {object }}, d_{\text {medium }}\right),
$$

where the OPD depends on the refractive index and thickness of both the biological sample and the material containing the object itself $[38-40,46]$. Due to their different optical path, the reference and object waves interfere at the surface of the recording device, and the hologram is proportional to the intensity of this interference pattern.

The resulting intensity distribution depends on the phase difference and contains information about the morphology of the sample under test. Therefore, the holographic approach employs the interference between the object and reference beams to codify the phase information to a recordable intensity distribution [38-40, 46]. Indeed, despite an optical field consists of amplitude and phase distributions, all detectors record intensity only. By using the information incorporated in the acquired hologram, a discrete version of the complex optical wavefront diffused by the sample object is numerically reconstructed at the plane of acquisition [38-40, 42-47].

The morphological analysis of the spermatozoa was carried out through the acquisition of a single-shot hologram (Figure 1(a)), corresponding to the intensity pattern of the interference between the object and the reference beams. The resulting intensity distribution depends on the phase difference and contains information about the morphology of the sample under test. We used the "off-axis" configuration, introducing a small angle between the object and reference beams in order to avoid the spatial overlapping of three diffraction terms [5]. Figure 1(b) shows the acquired reference hologram, and Figure 1(c) represents the reconstructed amplitude map at the acquisition plane. By numerically processing the amplitude map reported in Figure 1(c) with the refocusing algorithm [8,54], it is possible to provide the focused amplitude map of the propagated object field (Figure 1(d)).

2.3. Combined Raman/Holographic Microscope. The optical setup enabling simultaneous Raman and holographic imaging requires the use of two different laser sources $[8,36]$. The setup used for the sperm cell analysis is schematized in Figure 2 [8]. The Raman excitation was provided by a diode laser at $532 \mathrm{~nm}$ (Laser Quantum, Opus, max power $=2 \mathrm{~W}$ ). The green light was focused on the sample by an objective lens (OBJ, Olympus, 60x, 1.2 NA, water immersion). The back-scattered light, collected by the objective lens (OBJ), was reflected by a long-pass dichroic mirror (DM, with a cut frequency of $600 \mathrm{~nm}$, reflecting the radiation at $532 \mathrm{~nm}$ 


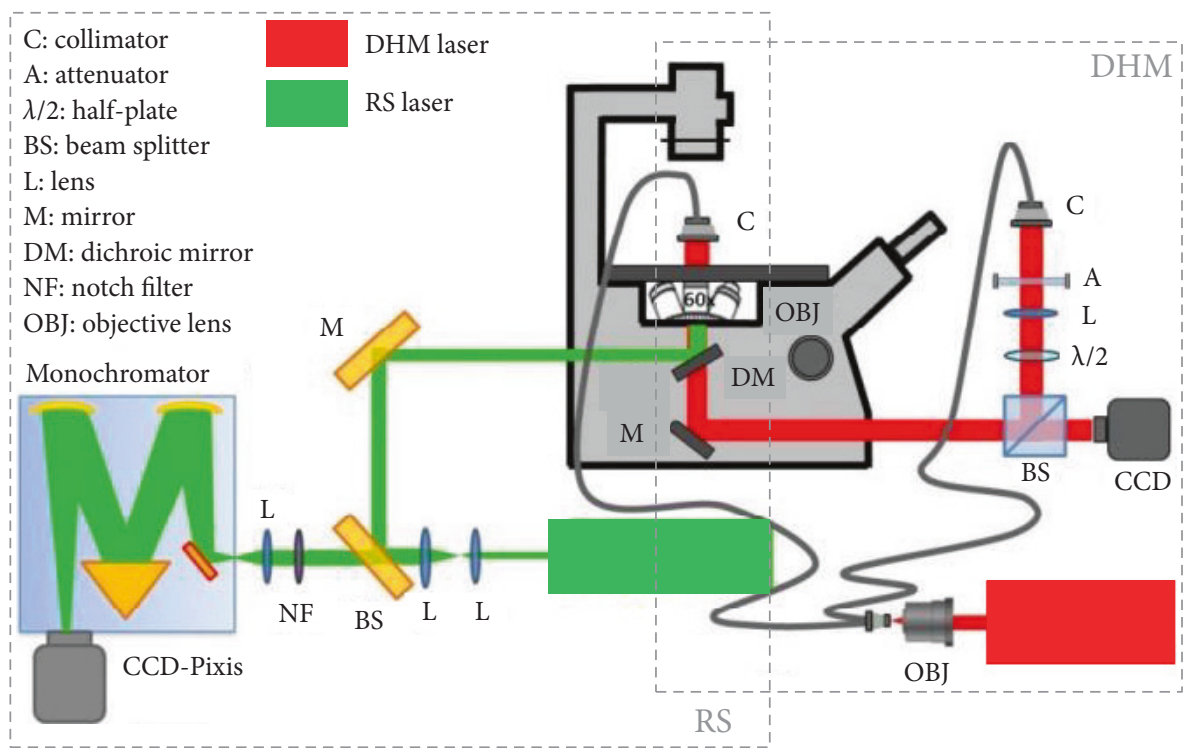

FIGURE 2: Experimental setup enabling the simultaneous digital holographic microscopy (DHM) and Raman spectroscopy (RS) measurements [8].

and being transparent in the spectral range above $600 \mathrm{~nm}$ ) and was separated from the holographic wavelength. The Raman-scattered light was reflected by a dichroic beam splitter (BS45; RazorEdge $45^{\circ}$ beamsplitter, Semrock) and the Rayleigh light was rejected. The Raman signal was additionally filtered using a laser-blocking filter (NF0; RazorEdge $0^{\circ}$ notch filter, Semrock) to eliminate the residual Rayleigh scattering and then focused onto the entrance slit of a monochromator set at $200 \mu \mathrm{m}$. The monochromator (S; Acton SP2300, Princeton Instruments) is equipped with a 1200 lines/mm holographic grating providing an estimated spectral resolution of approximately $2 \mathrm{~cm}^{-1}$. The Raman signal is finally detected by using a back-illuminated CCD camera (CCD2; PIXIS:400BR-eXcelon CCD, Princeton Instruments), thermoelectrically cooled at $-70^{\circ} \mathrm{C}$.

Different configurations have been proposed for acquiring Raman maps: point, line-scanning, or wide-field Raman imaging systems [55]. Pavillon et al. proposed the use of two galvanomirrors to scan the beam and capture a twodimensional data set containing all spectra of one spatial line, minimizing Raman imaging time [36]. In our recent paper, by exploiting the concept of orthogonal illuminating fields and using spatial light modulators, we realized a scan-free spontaneous Raman imaging system [53]. The sperm cell maps reported in this review have been acquired by scanning the sample using a motorized XY stage (stage travel $100 \mathrm{~mm} \times 120 \mathrm{~mm}$, steps $=50 \mathrm{~nm}$ ).

The holographic optical setup is basically an interferometer, and the hologram results from the interference of the object beam with the reference wave. Different optical setups have been proposed to perform microscopy with holography $[38,56,57]$. For example, a lens-free and double-wavelength setup has been developed by Su et al. for tracking in real-time 3D sperm trajectories [48]. Merola et al., using optical tweezers to trap and rotate the cell sample, reconstructed a tomographic 3D model of the spermatozoon [45]. Typically, the sources used in holography are lasers; however, coherent beams are very sensitive to any defect in the optical paths and are affected by coherent noise, which reduces the optical quality of the reconstructed images. To ease the problem of coherent noise, the Dubois's group developed a digital holographic microscope that works in transmission with a partially coherent source [58]. The optical setup, used for the experiments reported in the review, was a typical holographic microscope configuration based on the use of a microscope objective, in which the sample is observed in transmission, offering the largest numerical apertures, the easiest combination with the Raman microscope $[8,36]$, and allowing to explore transparent specimen including living cells.

The holographic microscope requires the use of coherent illumination, which, in our case, was provided by an active locked single longitudinal mode cavity laser (Laser Quantum, Torus, $\lambda=660 \mathrm{~nm}$, max power $=200 \mathrm{~mW}$, coherence length $>100 \mathrm{~m}$ ). The laser beam was split into two beams: the object and reference beams. Importantly, the reference beam was controllable in intensity and polarization, enabling us to improve contrast and signal. The collimated object beam, transmitted by the sample, was collected by the objective lens (OBJ), separated from the Raman signal through the dichroic mirror (DM), and recombined to the reference beam. The recombined object and reference beams were filtered and sent to the CCD camera (CCD1, $1392 \times 1040$ pixels array; pixel dimensions $\Delta x=\Delta y=4.7 \mu \mathrm{m})$.

2.4. Sample Preparation. A detailed description of the sample preparation is reported in $[7,8]$. In this study, we have used both bovine (provided and prepared by the Institute "Lazzaro Spallanzani", Rivolta d'Adda, Italy) and human sperm cells (provided by Centro di Fecondazione Assistita (CFA), Naples, Italy). The spermatozoa were washed and separated from the seminal fluid, incubated in a fixing solution, and, finally, resuspended in phosphate-buffered saline (PBS). 


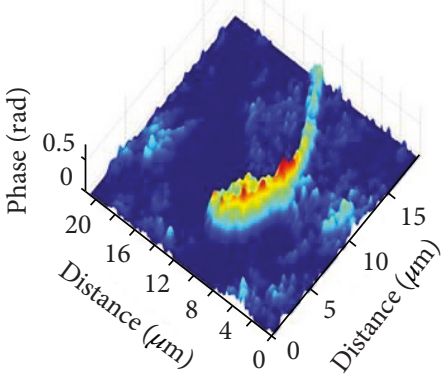

(a)

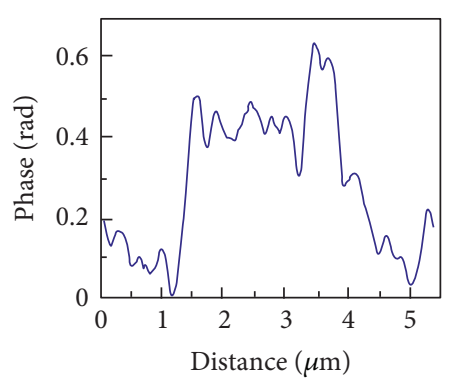

(b)

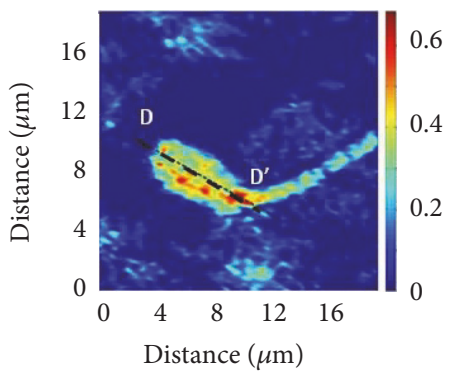

(c)

FIGURE 3: (a) Pseudo-3D representation of the phase map of the investigated spermatozoon obtained by digital holography microscopy. The arrow indicates the observed protuberance in the region connecting the tail to the head of the spermatozoon. (b) Phase intensity profile of the spermatozoon along the line $\mathrm{DD}^{\prime}$ stressed in (c) [7].

$1 \mu \mathrm{L}$ aliquot of the sperm suspensions was smeared onto a quartz coverslip air-dried and sealed on a quartz slide by using an ordinary "nail polish."

\section{Results}

3.1. Correlative Morphological and Physiological Characterization of Sperm Cell Defects. A powerful method for sperm cell assessment should provide morphological and physiological information on the analysed sample. For this purpose, in this section we correlate the holographic and Raman imaging approach.

The $3 \mathrm{D}$ reconstructed phase map is finally shown in Figure 3(a). Quantitative information on thickness can be obtained from the $3 \mathrm{D}$ image, by considering that each value of the digital holography map matrix represents the phase delay of the light passing through the sample that is related to the thickness and refractive index of the sperm cell [7]. The $3 \mathrm{D}$ image reconstruction highlighted the presence of a protuberance in the middle piece of the spermatozoon, indicated by the arrow in Figure 3(a). This alteration is clearly visible in the phase profile reported in Figure 3(b), associated to the line $\mathrm{DD}^{\prime}$ shown in the unwrapped phase-contrast map reconstruction of the spermatozoon reported in Figure 3(c) $[4,7]$. The morphological alteration observed in the $3 \mathrm{D}$ holographic map was confirmed by the images provided by the dual core confocal microscope $[4,7]$. However, in this latter case, a stack of about 40 images was required. Therefore, digital holography has the advantage to manage quantitative information, carrying out different numerical analysis such as area and volume estimation, profile along particular directions, and selection of different zones in one single acquisition [4-8].

In order to identify the biochemical nature of the protuberance, we have combined and correlated the digital holography results with the chemical images. A Raman spectrum is acquired at each and every pixel of the spermatozoon image and then interrogated to generate false colour images based on the molecular composition of the cell [53, 59]. The Raman microscope used for this experiment was an inverted confocal Raman microscope (XploRA, Jobin Yvon), equipped with a $532 \mathrm{~nm}$ laser source and described elsewhere [60]. A region of $9 \mu \mathrm{m} \times 9 \mu \mathrm{m}$ around the spermatozoon head was selected and imaged by scanning the interest region with steps of $0.2 \mu \mathrm{m}$, integration time per spectrum of $10 \mathrm{~s}$, and laser power on the sample of $1 \mathrm{~mW}$. Figure 4(a) shows the spectra acquired from different spermatozoon regions: the membrane (the green line), the nucleus (the red line), the acrosome (the blue line), and the middle piece (the darkyellow line). Pronounced Raman bands assigned to nucleic acids and DNA $\left(726,785,1095\right.$, and $\left.1581 \mathrm{~cm}^{-1}\right)$ identify the nucleus Raman spectrum.

The acrosomal vesicle spectrum reveals specific bands corresponding to proteins and lipids (1200-1300, 1480, and $\left.1600-1680 \mathrm{~cm}^{-1}\right)$. The membrane spectrum shows pronounced bands in the region between 1200 and $1400 \mathrm{~cm}^{-1}$ assigned to the presence of lipid and proteins. Finally, the middle piece Raman spectrum is characterized by strong peaks at 1005 and $1450 \mathrm{~cm}^{-1}$ consistent with the presence of proteins in this spectral region [7]. In Table 1, the Raman bands assigned to different molecular structures are reported [32]. Figure 4(b) shows the spermatozoon 


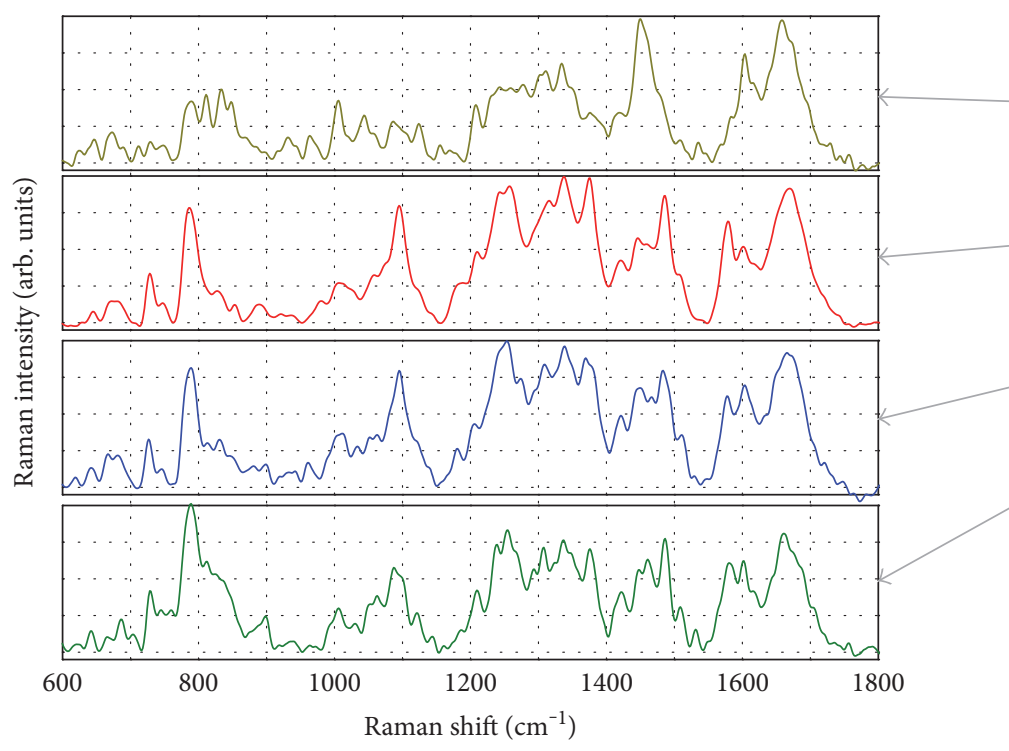

(a)

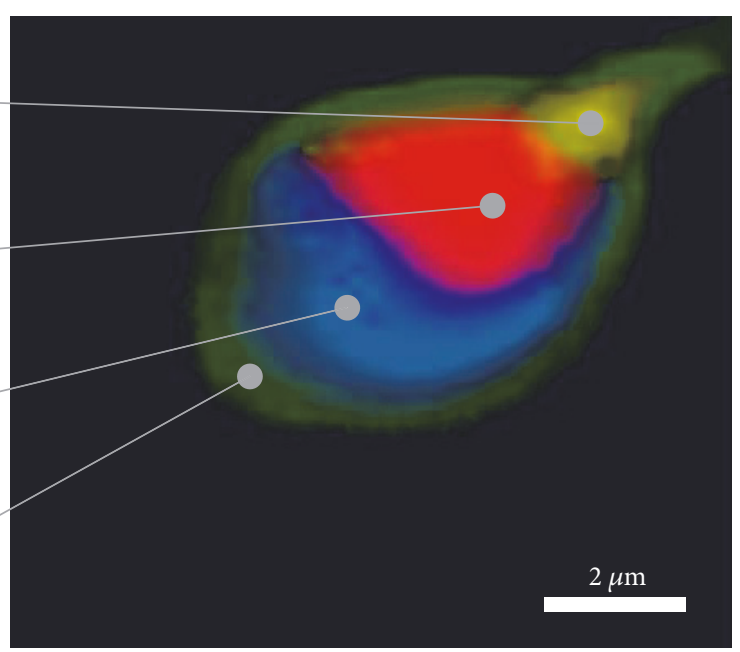

(b)

FIGURE 4: (a) Raman spectra (integration time: 10s) acquired from cell membrane (green line), acrosomal vesicle (blue line), nucleus (red line), and middle piece (dark-yellow line). The spectra have been used to reconstruct the false colour Raman image reported in (b) [7].

TABle 1: Raman band assignment to specific biochemical components.

\begin{tabular}{lc}
\hline Raman band $\left(\mathrm{cm}^{-1}\right)$ & Peak assignment \\
\hline 638 & Cytosine \\
665 & Thymine \\
681 & Guanine \\
726 & Adenine \\
751 & \\
785 & Thymine, cytosine, DNA bk \\
$810-900$ & Phenylalanine \\
1001 & CO \\
1053 & PO ${ }^{-}$bk \\
1095 & Proteins, $=\mathrm{CH}$ \\
1335 & Adenine, CH def \\
1375 & Thymine, adenine, guanine \\
1446 & Adenine, guanine, $\mathrm{CH} 2$ \\
$1500-1600$ & Adenine, guanine, cytosine, thymine, \\
$1600-1700$ & CH, CH2 \\
\hline
\end{tabular}

pseudocolour image reconstructed by associating a different colour to each Raman spectrum class. The chemical image confirmed the presence of the protuberance highlighted through digital holography in the middle piece region of the sperm cell. The Raman spectrum of the middle piece region was characterized by intense and sharp peaks assigned to the proteins $\left(1005\right.$ and $\left.1450 \mathrm{~cm}^{-1}\right)$, suggesting a correlation between the presence of the protuberance and the increase of the total amount of protein in that region [7].

3.2. Raman Spectroscopy and Holographic Microscopy for Sperm Cell Sex Sorting. What is needed clinically to study male reproductive function is a noninvasive, nondestructive analytical technique that provides specific information on the morphological and physiological quality of the cell. To demonstrate the sorting accuracy of our approach in providing precise and accurate information on the DNA quality of a specific sperm, we used the holographic/Raman method to identify X- and Y-bearing bovine sperm cells.

A direct method for sex predetermination in animals is based on the identification and sorting of X- and Y-sperm cells. Then, sex-sorted sperm cells can be used in conjunction with the artificial insemination or in vitro fertilization to produce the offspring of the desired sex. The main difference between $\mathrm{X}$ - and Y-sperm cells is due to the presence of the $\mathrm{X}$ - and $\mathrm{Y}$-chromosomes. Indeed, sex sorting can be performed by analysing the cell head size and volume, being the X-chromosome larger than the Y-chromosome, or the DNA cell content. In this section, we present a correlative holographic/Raman-based method for selective and sensitive discrimination of X- and Y-bearing bovine sperm cells considering both parameters: cell volume and DNA content.

By acquiring a single hologram, it is possible to reconstruct the phase map of a spermatozoon and calculate its thickness and, therefore, its head volume [7]. Figure 5 shows the main steps of the numerical procedure used to calculate the volume of X-and Y-bearing sperm heads. A MATLAB algorithm was implemented in order to evaluate the sperm head area. The reconstructed phase map was first zoomed in and cut. The interest region was isolated from the background by means of the Otsu's threshold method [61]. Then, the boundary of the selected region was determined, and its polygonal interpolation, filling, and expansion were performed in order to estimate the area of the sperm head. Finally, the head volume was calculated as the product of the phase map and the estimated area (Figure 5(d)) and by considering a uniform refractive index of 1.35 


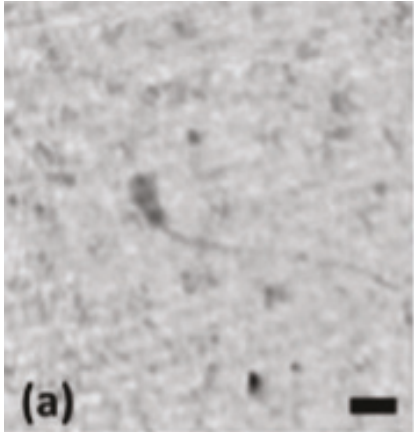

(a)

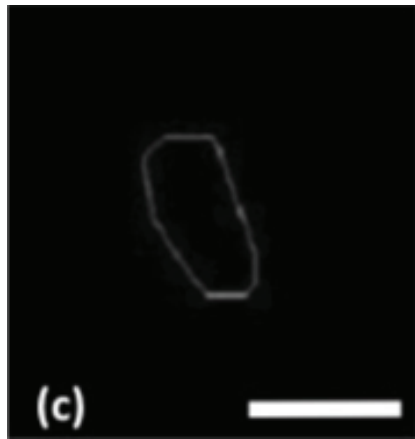

(c)

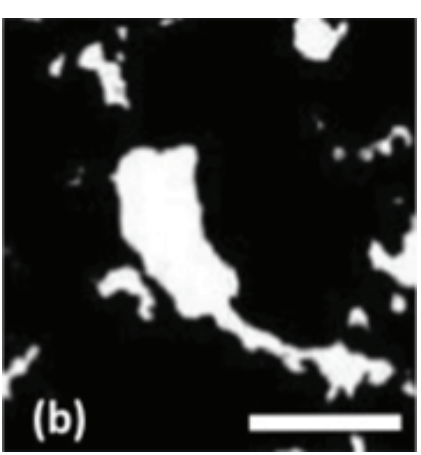

(b)

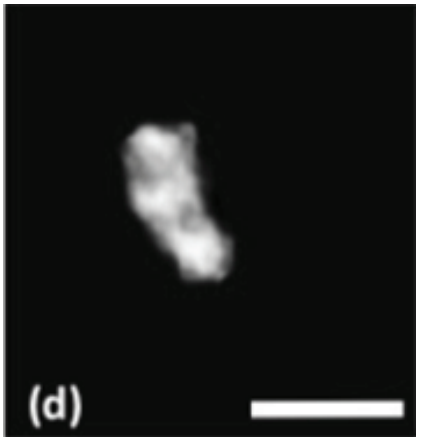

(d)

Figure 5: Volume estimation procedure from the phase map. (a) Phase map. (b) Selected head region by Otsu's threshold method. (c) Polygonal interpolation. (d) Final estimated sperm head volume. Scale bars: $5 \mu \mathrm{m}$ [7].

[4]. The cell head volume estimation procedure has been performed on $300 \mathrm{X}$ - and $300 \mathrm{Y}$-bearing sperm cells from three different bulls to take into account the variation from cell to cell and bull to bull. The results of the morphological analysis are summarized in Figure 6. The mean head volume ( \pm the standard error) of the X-bearing spermatozoa $\left(V_{x}=115 \pm 2 \mu \mathrm{m}^{3}\right)$ was slightly larger than that of the Y-bearing spermatozoa $\left(V_{y}=108 \pm 3 \mu \mathrm{m}^{3}\right)$, and the volume variation (about $\Delta V=6.0 \pm 2.5 \%$ ) is comparable to the expected differences in DNA content (3.9\% in bull) [6]. However, as shown in Figure 6, the two volume distributions are overlapped and the standard error of the volume variation is comparable to volume variation itself. Consequently, the quantitative morphological analysis performed by digital holography does not allow to unequivocally separate the two cell populations.

We additionally used the Raman approach for the labelfree identification of DNA content for X- or Y-bearing sperm cells and better separating the two cell populations [6]. 300 Raman spectra were acquired from each cell population with 20 s integration time and the experiments have been repeated on three different bulls. The sample autofluorescence was bleached, exposing the cell to the laser light for about $40 \mathrm{~s}$ before the spectral acquisition. The spectra were averaged, baseline corrected, smoothed, and normalized. Figure 7(a) shows typical Raman spectra in the fingerprint region, between 600 and $1800 \mathrm{~cm}^{-1}$, of X- (purple line) and Y- (blue line) bearing sperm cells of a bull acquired from the nucleus region. The main spectral differences were found in the peaks assigned to DNA nitrogenous bases $(726,785$, and

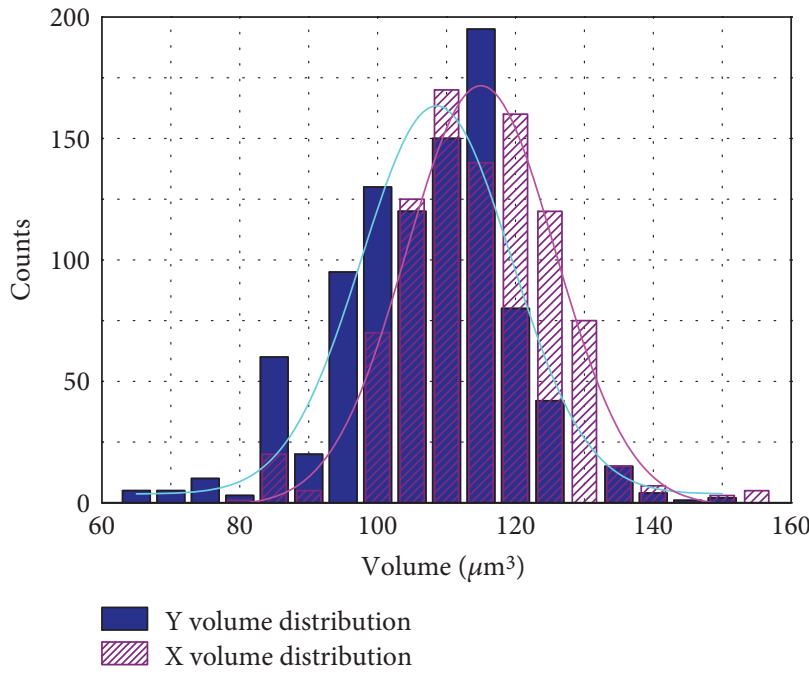

Figure 6: Volume distributions for X- (purple) and Y- (blue) bearing spermatozoa heads. The Gaussian fit for the two volume distributions is shown.

$\left.1581 \mathrm{~cm}^{-1}\right)$ and DNA backbone $\left(1095 \mathrm{~cm}^{-1}\right)$. The increased intensity of the peaks in X-sperm spectrum can be attributed to slightly higher DNA concentration in X- than in Y-bearing sperm cells. An additional difference between the two population of spectra was observed in the protein region (between 1400 and $1600 \mathrm{~cm}^{-1}$ ), probably due to the presence of HY antigen absent on the membrane of $\mathrm{X}$ - 


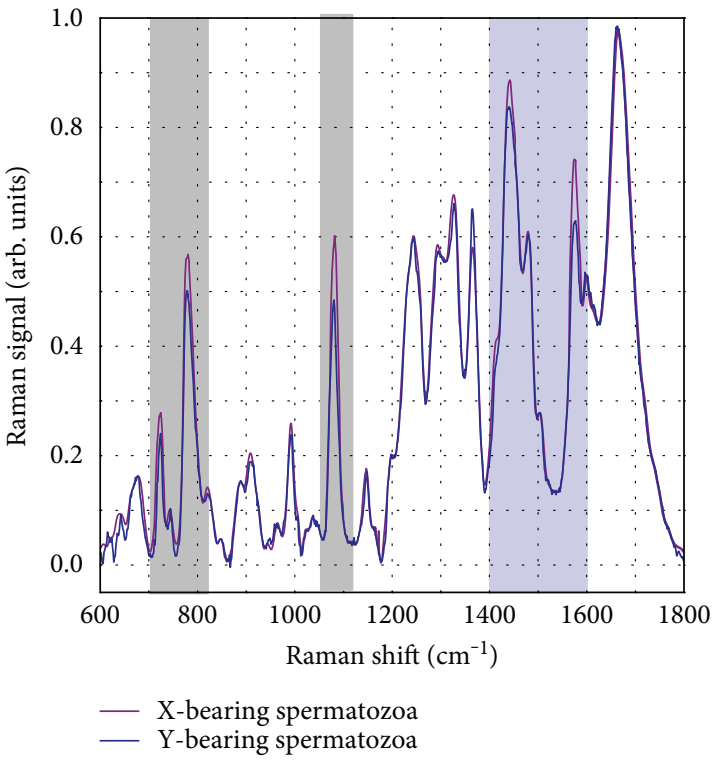

(a)
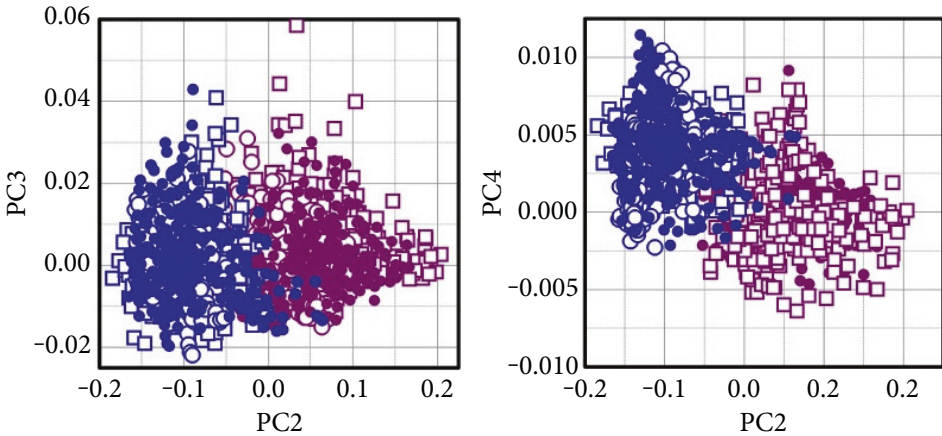

(c)

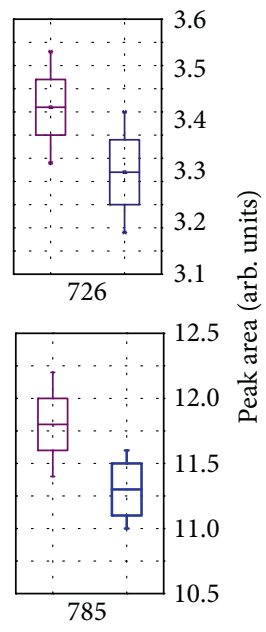

(b)

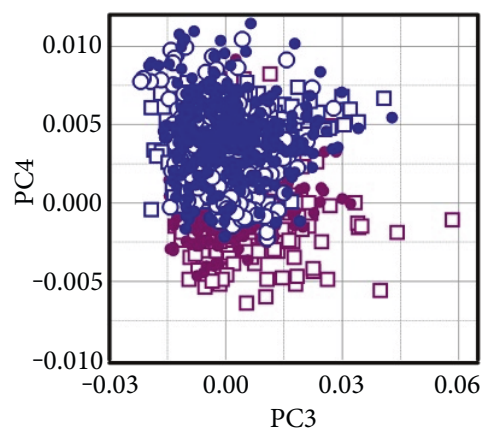

\begin{tabular}{lcc} 
& \multicolumn{2}{c}{ Predicted class } \\
\cline { 2 - 3 } Actual & X-spermatozoa & Y-spermatozoa \\
class & 822 & 78 \\
\hline X-spermatozoa & 91 & 809 \\
Y-spermatozoa & & \\
\hline
\end{tabular}

(d)

Figure 7: (a) Average Raman spectra of 900 X- (purple line) and 900 Y- (blue line) sperm cell spectra acquired in the "fingerprint" spectral region. The experiment has been performed on three different bulls. (b) Measured peak area of the bands at 726 and $785 \mathrm{~cm}^{-1}$, highlighting the different DNA contents measured for X- and Y-sperm cells; $\triangle A=4.2 \pm 0.9 \%$. (c) PCA score plots of PC2, PC3, and PC4 showing the separation of data corresponding to X-and Y-sperm cells. [6]. (d) Confusion matrix summarizing the correct and incorrect classifications for the analysed X-and Y-sperm spectra $[6,63,64]$.

sperm cells. By measuring the Raman band areas under the peaks at 726 and $785 \mathrm{~cm}^{-1}$ for the two cell populations (see histograms of Figure 7(b)), we found an area variation of $\Delta A=4.2 \% \pm 0.9 \%$, in good agreement with the expected differences in DNA content.

To evaluate the efficiency of the Raman approach for identification of the $\mathrm{X}$ - and Y-chromosome spermatozoa, we used the principal component analysis (PCA) [6, 7]. PCA is a multivariate statistical method that transforms the spectral data to a new coordinate system such that the greatest variance by any data projection comes to lie on the first coordinate (the first principal component, PC1), the second greatest variance on the second coordinate (PC2), and so on. By considering only few principal components, it is possible to reduce the data dimensionality and visualize and process high-dimensional data sets. By selecting the principal components PC2, PC3, and PC4 (providing the $60 \%$ of all spectral variability), we find the three-dimensional space through the high-dimensional data set in which the data is most spread out (see Figure 7(c)) and each cell type is clearly 


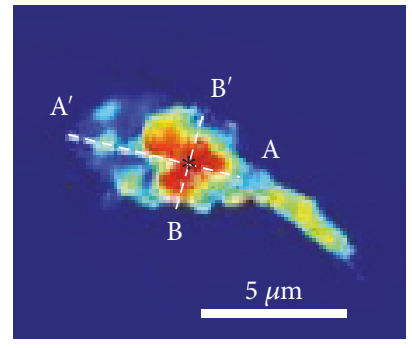

(a)

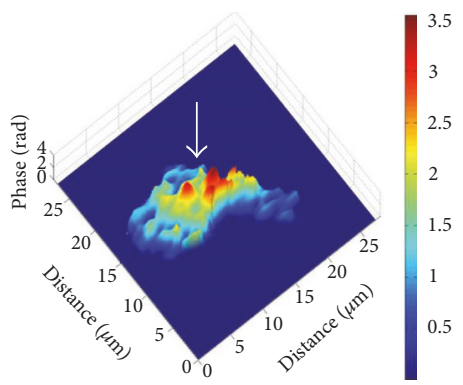

(e)

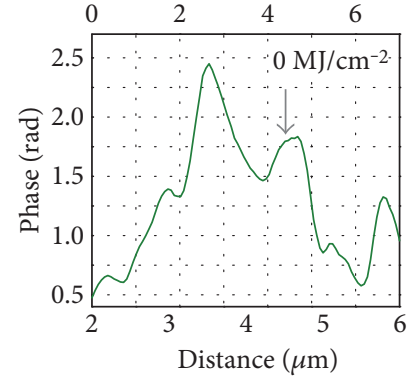

(b)

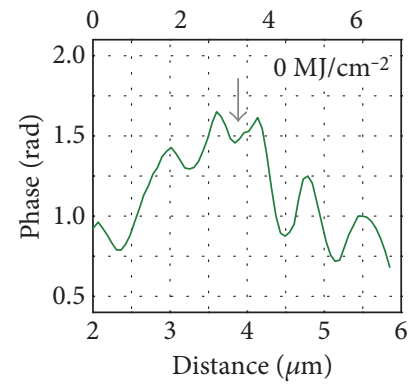

(f)

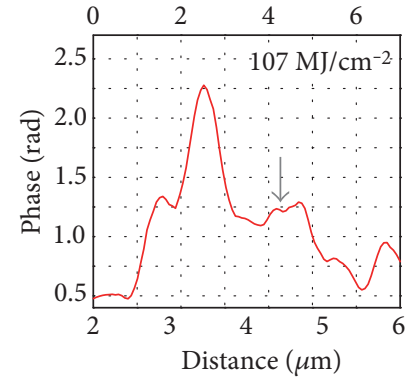

(c)

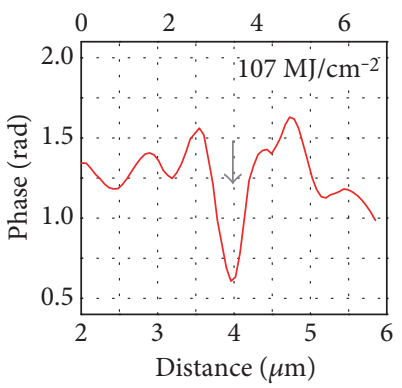

(g)

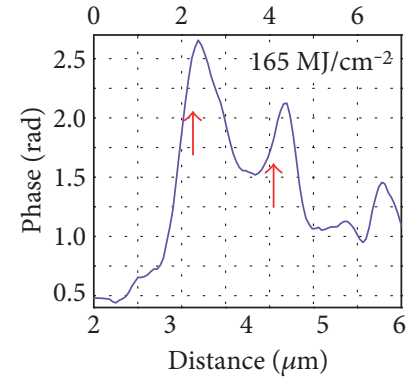

(d)

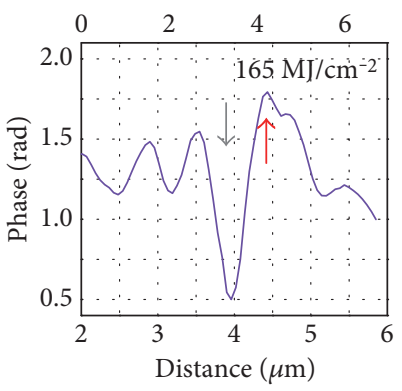

(h)

FIGURE 8: (a) Phase map of the sperm cell region reconstructed at the focus plane; the phase profile along the lines $\mathrm{BB}^{\prime}$ (b-d) and $A A^{\prime}$ (f-h) and the Raman spectra in the point $\left({ }^{*}\right)$ are monitored during the laser exposition. (e) $3 \mathrm{D}$ representation of the phase map of the investigated spermatozoon obtained by digital holography microscopy [8].

separated from the other one as indicated by the different marked and coloured scores. The first principal component PC1 (accounting for the 38\% variance) is not included in the data classification, as essentially due to the background variation and not directly attributed to the cell differences [6]. The PCA has been additionally used in a predictive way, by building up the confusion matrix, shown in Figure $7(\mathrm{~d})$, using the leave-one-out classification approach $[62,63]$. The confusion matrix summarizes that 1631 out of 1800 acquired spectra are correctly classified, providing a high prediction accuracy of $90.6 \%$.

\subsection{Simultaneous Anatomical and Biochemical Analysis of} Sperm Cell Photodamage. The low laser intensities and label-free nature of the proposed Raman/holographic approach should prevent semen damage after sorting, without affecting the discrimination accuracy. To demonstrate the nondestructive ability of our photonic approach for the used laser powers, we investigated the morphological and physiological damage induced by the laser light by using the combined and simultaneous approach based on digital holography and Raman spectroscopy techniques [8]. To simultaneously acquire the Raman spectrum and the hologram of the selected sperm cell, we used a single objective lens (OBJ2 in Figure 2) for both red and green light. In this experimental configuration, by focusing the Raman laser on the sample (green laser), the holographic image recorded by using the coherent red light resulted out of its focal plane (Figure 1). However, the digital holographic approach combined with the double exposure technique powerfully allows to numerically recovering the correct focus plane and remove the optical aberrations $[7,8,54]$. Basically, the first acquisition is performed by illuminating the sample region (Figure 1(a)), while the second one by illuminating a reference-free region nearby the sample (Figure 1(b)). The second hologram contains all the required information about the aberrations introduced by the optical components, including the defocusing [4-8]. Therefore, it is possible to compensate these aberrations and numerically propagate the object optical field [64].

By using this experimental approach, we studied the effect of the green laser light (Raman probe) by irradiating a specific region of the sperm cell and increasing the power from $4.4 \mathrm{~mW}$ to $50 \mathrm{~mW}$. A high numerical aperture microscope objective (NA 1.2) has been used to focus the green laser beam into a spot of about $0.36 \mu \mathrm{m}$ in diameter, providing fluences in the range of $13-165 \mathrm{MJ} / \mathrm{cm}^{2}$. The red light used for holographic measurements was set at $100 \mathrm{~mW}$ (red light fluence of $100 \mathrm{~mJ} / \mathrm{cm}^{2}$ ). For the selected experimental conditions, any possible degradation effect associated to the red laser light can be neglected, even after hours of irradiation [65]. The postacrosomal sperm cell region was irradiated for $3 \mathrm{~s}$ and, after each exposure, a single hologram and Raman spectrum were simultaneously acquired.

Figure 8 shows the phase profile variations associated to the irradiating fluences, along two different sperm directions: the line $A^{\prime}$ (Figures $8(\mathrm{~b})-8(\mathrm{~d})$ ) and $\mathrm{BB}^{\prime}$ (Figures $8(\mathrm{f})-8(\mathrm{~h})$ ). The arrow highlights the spermatozoa regions where the most relevant morphological alterations were observed. At laser fluences greater than $40 \mathrm{MJ} / \mathrm{cm}^{2}$, we experimentally observed a progressive reduction in the height, and therefore in the volume of the cell as visible in Figures 8(c) and 8(g) (see grey arrows). The phase map 3D reconstruction of the irradiated spermatozoon, shown in Figure 8(e), clearly shows 


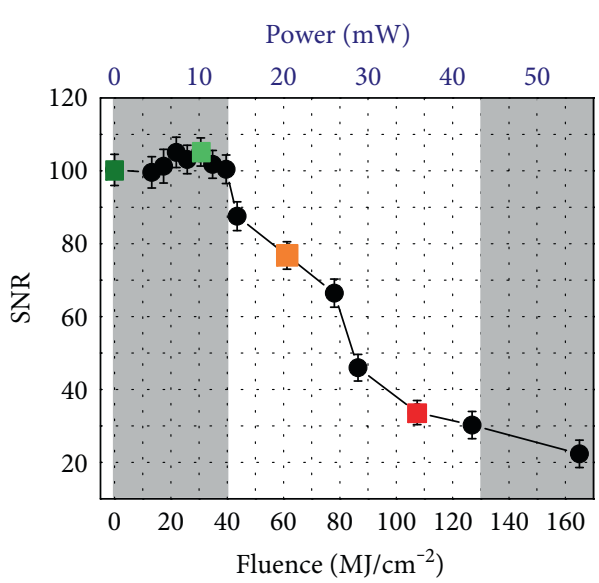

(a)

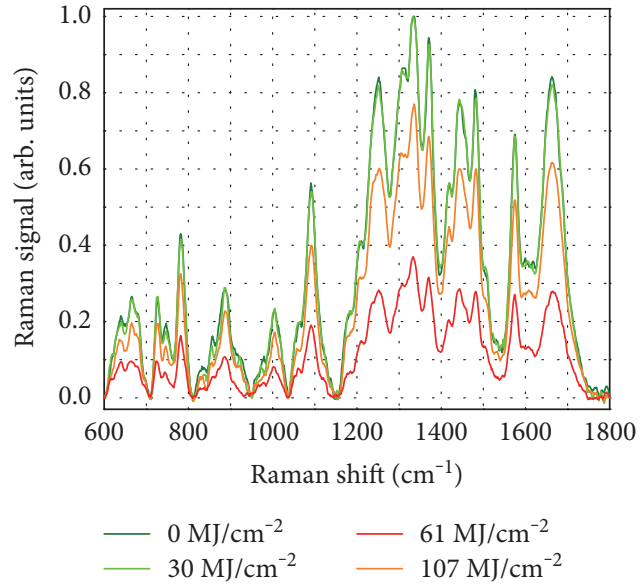

(b)

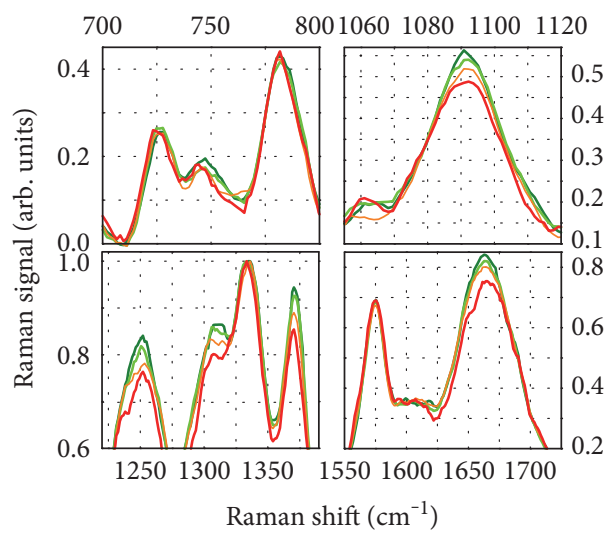

(c)

FIgURE 9: (a) Raman signal-to-noise ratio (SNR) measured as the ratio of the maximum Raman peak intensity at $1335 \mathrm{~cm}^{-1}$ to the standard deviation of the region between 1750 and $1800 \mathrm{~cm}^{-1}$ as function of the applied green light irradiation levels between 0 and $165 \mathrm{MJ} / \mathrm{cm}^{2}$. (b) Representative spectra relative to the laser fluences of $0,30,61$, and $107 \mathrm{MJ} / \mathrm{cm}^{2}$ are shown. (c) Zoom of the spectra in the DNA and protein regions [8].

the morphological photodamage induced by a green laser fluence of $107 \mathrm{MJ} / \mathrm{cm}^{2}$. On the other hand, at fluences higher than $130 \mathrm{MJ} / \mathrm{cm}^{2}$, the phase intensity profile along the line $\mathrm{BB}^{\prime}$ shows a slight increase of cell height on the left side (see red arrow in Figure 8) that could be explained with the internalization of biological material through the plasma membrane when the laser-exposed region was subjected to a sort of "photoporation." Indeed, by analysing Figure 8(d), the sperm cell seemed to swell. Finally, at fluences higher than $170 \mathrm{MJ} / \mathrm{cm}^{2}$, the spermatozoon is completely disintegrated.

We simultaneously acquired the Raman signal using a green laser power of $0.5 \mathrm{~mW}$ on the sample and an integration time of $20 \mathrm{~s}$ (green light fluence of $10 \mathrm{MJ} / \mathrm{cm}^{2}$ ) to study the physiological cell modification correlated to the morphological alteration. We measured the Raman signal-to-noise ratio (SNR) as the ratio of the maximum Raman peak intensity at $1335 \mathrm{~cm}^{-1}$ to the standard deviation of the region between 1750 and $1800 \mathrm{~cm}^{-1}$ and its variation as function of the applied green light irradiation levels (in terms of fluences and corresponding powers). As shown in Figure 9(a), in the fluence range of $0-40 \mathrm{MJ} / \mathrm{cm}^{2}$, the SNR remains almost constant; in the range $40-120 \mathrm{MJ} / \mathrm{cm}^{2}$, it decreases almost linearly with the laser fluences, and at fluences higher than $130 \mathrm{MJ} / \mathrm{cm}^{2}$, the SNR riches a plateau. In Figure 9(b), three more representative Raman spectra corresponding to the unexposed, $61 \mathrm{MJ} / \mathrm{cm}^{2}$, and $107 \mathrm{MJ} / \mathrm{cm}^{2}$ fluence are shown. In order to allow the comparison between the data, each spectrum was normalized to the maximum peak and then divided by the standard deviation. The Raman spectra confirm the degradation effect at irradiation fluences above the threshold of $40 \mathrm{MJ} / \mathrm{cm}^{2}$. At fluences higher than $130 \mathrm{MJ} / \mathrm{cm}^{2}$, an increase of the luminescence background of the Raman signal was observed confirming a local heating of the sample [65] due to the absorption at $532 \mathrm{~nm}$ [66-70] and a possible internalization of biological material. Additional considerations can be done analysing the relative Raman peak variations for fluences lower than $40 \mathrm{MJ} / \mathrm{cm}^{2}$.

As shown in Figure 9(c), the Raman bands related to localized vibration of the DNA bases $\left(700-800 \mathrm{~cm}^{-1}\right)$ remains almost invariant when irradiated by the green laser light, while the Raman bands associated to OPO backbone $\left(900-1100 \mathrm{~cm}^{-1}\right)$ are subjected to photoinduced oxidation and the peak at $1095 \mathrm{~cm}^{-1}$, decreases in intensity proportionally to the break of the double-helical structure with the 
fluence increase [71]. More precisely, it was observed that a relative intensity decreases already after $30 \mathrm{MJ} / \mathrm{cm}^{2}$. In the spectral range $1200-1400 \mathrm{~cm}^{-1}$, an intensity reduction of the Raman bands at 1250 and $1375 \mathrm{~cm}^{-1}$ is already visible for fluence of $30 \mathrm{MJ} / \mathrm{cm}^{2}$, probably due to alterations in the secondary and tertiary conformation of the proteins. Finally, all native nucleic acids exhibit a broad and intense band near $1668 \mathrm{~cm}^{-1}$, which originates from coupled $\mathrm{C}=\mathrm{O}$ stretching and $\mathrm{N}-\mathrm{H}$ deformation modes (see Table 1) that is highly sensitive to disruption of Watson-Crick hydrogen bonding $[72,73]$ as shown in Figure 9(c).

\section{Discussion and Conclusions}

In this work, a promising optical approach, based on digital holography and Raman spectroscopy technologies, has been proposed for the quality assessment of sperm cells. The great advantage of digital holography is the possibility to retrieve $3 \mathrm{D}$ quantitative imaging of the sample under investigation with a single-shot acquisition and directly in its native environment [4-8]. Raman spectroscopy, based on the inherent molecular vibration excitation/ response, allows label-free, non-destructive (taking into account the laser wavelength and power) biochemical investigations.

Applying the holographic approach for analysing the sperm cells, high-resolution images have been obtained, clearly highlighting some morphological alterations. In particular, a sort of "protuberance" was observed in the postacrosomal region of few investigated spermatozoa and was correlated, by using the Raman imaging approach, to the increased protein concentration (probably due to the presence of centrioles) [7]. Therefore, with the proposed combined approach, it is possible nondestructively delineating the distribution of DNA and protein in the head, acrosome, and tail but also detecting morphologically and physiologically small discrepancies such as the presence of defects in a correlative manner. It provides information at the molecular level, allowing investigation of functional groups, bonding types, and molecular conformations and correlating them to the cell structural properties.

Spectral bands in vibrational spectra are specific to each molecule and give direct information about the biochemical composition and have been used to noninvasively discriminate between $\mathrm{X}$ - and $\mathrm{Y}$-bearing bovine sperm cells by analysing the DNA content, plasma membrane proteins, or biochemical signatures with high accuracy, completing the morphological information (volume measurements) provided by the holographic imaging. Therefore, it could be a very promising method to test the functionality of preselected sperm samples due to its high chemical identification capabilities and as a noninvasive optical method.

Sperm sample chemical assessment is possible by several methods [74-78]. However, none of the current available techniques are noninvasive or nondamaging. Therefore, we assessed doses and risks of exposing sperm cells to the excitation laser light during Raman/holographic measurements. In this case, digital holography and Raman spectroscopy simultaneous approach was used for studying the photodamage induced by visible green light in the spermatozoa structure. DNA and proteins resulted the more affected biochemical constituents under visible green light irradiation, already for fluence of $30 \mathrm{MJ} / \mathrm{cm}^{2}$ (laser power $10 \mathrm{~mW}$ ), where no morphological changes have been detected by the holographic characterization. This result confirms that there is not a direct relationship between the morphological aspects of the spermatozoon and the integrity of its biochemical structures. That is the reason why an efficient sperm analysis cannot be based only on the morphological criterion but it has to be accompanied and completed with a biochemical characterization, as that provided by Raman spectroscopy. Reducing the laser power to 1-2 $\mathrm{mW}$, we found no evidence for detrimental effects, as DNA fragmentation, membrane damage, and protein alteration, exposing the spermatozoa to the laser light. This opens up possibilities to investigate a multitude of morphological and physiological parameters, for example, DNA fragmentation and presence of vacuole, under near-physiological conditions.

One major limitation is the long acquisition time required to construct the Raman image. Several approaches can be used to overcome this limitation providing a faster imaging modality and allowing investigation of several sperm cells simultaneously, these include

(i) the use of galvanomirrors to scan the beam and capture a two-dimensional data set containing all spectra of one spatial line [36],

(ii) the use of spatial light modulators and orthogonal illuminating fields to realize a scan-free spontaneous Raman imaging system [53],

(iii) the use of a faster Raman-based imaging approach, such as CARS microscopy [79-84] or SERS microscopy [29, 85-90], providing chemical images with large fields of view at a video rate.

An important consideration to be done concerns the costs and feasibility. Although instrumentation for Raman spectroscopy is fairly simple, indeed portable Raman spectrometers are already available; the combined approach is expensive requiring the use of lasers, "open microscopes," and specialized operators.

Additionally, the performance of a $\mathrm{DH}$ system is related to its resolution that is determined by the magnification of the objective lens and by the finite size of the pixels of the CCD due to the finite extent of the camera itself [91].

In conclusion, the proposed Raman/holography imaging approach could be a very promising method to test the physiology and morphology of preselected sperm cells due its noninvasive and nondestructive nature and its high-specific identification capabilities. This would be crucial for the investigation of human sperm before assisted fecundation, where $100 \%$ selection success is mandatory. However, more investigations on living sperm cells closer to the physiological conditions are required in order to validate our results for effective biomedical applications. 


\section{Conflicts of Interest}

The authors declare that there is no conflict of interest regarding the publication of this paper.

\section{Acknowledgments}

The authors acknowledge Roberto Puglisi, Donatella Balduzzi, and Andrea Galli, from Instituto Sperimentale Italiano "Lazzaro Spallanzani”, Rivolta d'Adda (CR), Italy, and Gianfranco Coppola and Brian Dale from CFA (Centro di Fecondazione Assistita), Naples, Italy, for providing the samples.

\section{References}

[1] C. L. Barratt, "Semen analysis is the cornerstone of investigation for male infertility," The Practitioner, vol. 251, no. 1690, p. 8, 2007.

[2] S. Cayli, A. Jakab, L. Ovari et al., "Biochemical markers of sperm function: male fertility and sperm selection for ICSI," Reproductive Biomedicine Online, vol. 7, no. 4, pp. 462-468, 2003.

[3] T. G. Cooper, E. Noonan, S. von Eckardstein et al., "World Health Organization reference values for human semen characteristics," Human Reproduction Update, vol. 16, no. 3, pp. 231-245, 2009.

[4] G. Di Caprio, M. A. Gioffre, N. Saffioti et al., "Quantitative label-free animal sperm imaging by means of digital holographic microscopy," IEEE Journal of Selected Topics in Quantum Electronics, vol. 16, no. 4, pp. 833-840, 2010.

[5] G. Di Caprio, M. A. Ferrara, L. Miccio et al., "Holographic imaging of unlabelled sperm cells for semen analysis: a review," Journal of Biophotonics, vol. 8, no. 10, pp. 779-789, 2015.

[6] A. C. De Luca, S. Managó, M. A. Ferrara et al., "Non-invasive sex assessment in bovine semen by Raman spectroscopy," Laser Physics Letters, vol. 11, no. 5, p. 055604, 2014.

[7] M. A. Ferrara, G. Di Caprio, S. Managò et al., "Label-free imaging and biochemical characterization of bovine sperm cells," Biosensors, vol. 5, no. 2, pp. 141-157, 2015.

[8] M. A. Ferrara, A. De Angelis, A. C. De Luca, G. Coppola, B. Dale, and G. Coppola, "Simultaneous holographic microscopy and Raman spectroscopy monitoring of human spermatozoa Photodegradation," IEEE Journal of Selected Topics in Quantum Electronics, vol. 22, no. 3, pp. 1-8, 2016.

[9] B. Bartoov, A. Berkovitz, and F. Eltes, "Selection of spermatozoa with normal nuclei to improve the pregnancy rate with intracytoplasmic sperm injection," The New England Journal of Medicine, vol. 345, no. 14, pp. 1067-1068, 2001.

[10] B. Bartoov, A. Berkovitz, and F. Eltes, "Relationship between human sperm subtle morphological characteristics and IVFICSI outcome," Journal of Andrology, vol. 23, pp. 1-8, 2002.

[11] C. Celik-Ozenci, A. Jakab, T. Kovacs et al., "Sperm selection for ICSI: shape properties do notpredict the absence or presence of numericalchromosomal aberrations," Human Reproduction, vol. 19, no. 9, pp. 2052-2059, 2004.

[12] R. Sharma, J. Masaki, and A. Agarwal, "Sperm DNA fragmentation analysis using the TUNEL assay," Methods in Molecular Biology, vol. 927, pp. 121-136, 2013.

[13] L. Simon, D. Lutton, J. McManus, and S. E. Lewis, "Sperm DNA damage measured by the alkaline comet assay as an independent predictor of male infertility and in vitro fertilization success," Fertility and Sterility, vol. 95, no. 2, pp. 652-657, 2011.

[14] J. L. Fernández, L. Muriel, V. Goyanes et al., "Simple determination of human sperm DNA fragmentation with an improved sperm chromatin dispersion test," Fertility and Sterility, vol. 84, no. 4, pp. 833-842, 2005.

[15] D. P. Evenson, "Sperm chromatin structure assay (SCSA $\left.{ }^{\circledR}\right)$," Spermatogenesis Methods Protocol, vol. 927, pp. 147-164, 2013.

[16] V. Sánchez, J. Wistuba, and C. Mallidis, "Semen analysis: update on clinical value, current needs and future perspectives," Reproduction, vol. 146, no. 6, pp. R249-R258, 2013.

[17] R. Henkel, E. Kierspel, M. Hajimohammad et al., "DNA fragmentation of spermatozoa and assisted reproduction technology," Reproductive Biomedicine Online, vol. 7, no. 4, pp. 477-484, 2003.

[18] G. Rusciano, A. C. De Luca, A. Sasso, and G. Pesce, "Enhancing Raman tweezers by phase-sensitive detection," Analytical Chemistry, vol. 79, no. 10, pp. 3708-3715, 2007.

[19] G. Rusciano, A. C. De Luca, G. Pesce, and A. Sasso, "On the interaction of nano-sized organic carbon particles with model lipid membranes," Carbon, vol. 47, no. 13, pp. 2950 2957, 2009.

[20] A. C. De Luca, K. Dholakia, and M. Mazilu, "Modulated Raman spectroscopy for enhanced cancer diagnosis at the cellular level," Sensors, vol. 15, no. 6, pp. 13680-13704, 2015.

[21] S. Managò, C. Valente, P. Mirabelli et al., "A reliable Ramanspectroscopy-based approach for diagnosis, classification and follow-up of B-cell acute lymphoblastic leukemia," Scientific Reports, vol. 6, article 24821, 43 pages, 2016.

[22] P. C. Ashok, A. C. De Luca, M. Mazilu, and K. Dholakia, "Enhanced bioanalyte detection in waveguide confined Raman spectroscopy using wavelength modulation," Journal of Biophotonics, vol. 4, no. 7-8, pp. 514-518, 2011.

[23] M. Harz, P. Rösch, and J. Popp, "Vibrational spectroscopy-a powerful tool for the rapid identification of microbial cells at the single-cell level," Cytometry. Part A, vol. 75, no. 2, pp. 104-113, 2009.

[24] D.-L. Chen, N. Li, L. Lin et al., "Confocal mirco-Raman spectroscopic analysis of the antioxidant protection mechanism of the oligosaccharides extracted from Morinda officinalis on human sperm DNA," Journal of Ethnopharmacology, vol. 153, no. 1, pp. 119-124, 2014.

[25] K. Meister, D. A. Schmidt, E. Bründermann, and M. Havenith, "Confocal Raman microspectroscopy as an analytical tool to assess the mitochondrial status in human spermatozoa," Analyst, vol. 135, no. 6, pp. 1370-1374, 2010.

[26] E. Edengeiser, K. Meister, E. Bründermann, S. Büning, S. Ebbinghaus, and M. Havenith, "Non-invasive chemical assessment of living human spermatozoa," RSC Advances, vol. 5, no. 14, pp. 10424-10429, 2015.

[27] T. Huser, C. A. Orme, C. W. Hollars, M. H. Corzett, and R. Balhorn, "Raman spectroscopy of DNA packaging in individual human sperm cells distinguishes normal from abnormal cells," Journal of Biophotonics, vol. 2, no. 5, p. 322, 2009.

[28] C. Mallidis, J. Wistuba, B. Bleisteiner et al., "In situ visualization of damaged DNA in human sperm by Raman microspectroscopy," Human Reproduction, vol. 26, no. 7, pp. 1641-1649, 2011.

[29] A. C. De Luca, P. Reader-Harris, M. Mazilu, S. Mariggio, D. Corda, and A. Di Falco, "Reproducible surface-enhanced 
Raman quantification of biomarkers in multicomponent mixtures," ACS Nano, vol. 8, no. 3, pp. 2575-2583, 2014.

[30] M. Li, J. Xu, M. Romero-Gonzalez, S. A. Banwart, and W. E. Huang, "Single cell Raman spectroscopy for cell sorting and imaging," Current Opinion in Biotechnology, vol. 23, no. 1, pp. 56-63, 2012.

[31] H. Abramczyk, J. Surmacki, M. Kopeć, A. K. Olejnik, A. Kaufman-Szymczyk, and K. Fabianowska-Majewska, "Epigenetic changes in cancer by Raman imaging, fluorescence imaging, AFM and scanning near-field optical microscopy (SNOM). Acetylation in normal and human cancer breast cells MCF10A, MCF7 and MDA-MB-231," Analyst, vol. 141, no. 19, pp. 5646-5658, 2016.

[32] I. Notingher, "Raman spectroscopy cell-based biosensors," Sensors, vol. 7, no. 8, pp. 1343-1358, 2007.

[33] N. Li, D. Chen, Y. Xu, S. Liu, and H. Zhang, "Confocal Raman micro-spectroscopy for rapid and label-free detection of maleic acid-induced variations in human sperm," Biomedical Optics Express, vol. 5, no. 5, pp. 1690-1699, 2014.

[34] V. Sánchez, K. Redmann, J. Wistuba et al., "Oxidative DNA damage in human sperm can be detected by Raman microspectroscopy," Fertility and Sterility, vol. 98, no. 5, pp. 11241129, 2012.

[35] F. Liu, Y. Zhu, Y. Liu et al., "Real-time Raman microspectroscopy scanning of the single live sperm bound to human zona pellucida," Fertility and Sterility, vol. 99, no. 3, pp. 684-689, 2013.

[36] N. Pavillon, A. J. Hobro, and N. I. Smith, "Cell optical density and molecular composition revealed by simultaneous multimodal label-free imaging," Biophysical Journal, vol. 105, no. 5, pp. 1123-1132, 2013.

[37] P. Marquet, B. Rappaz, P. J. Magistretti et al., "Digital holographic microscopy: a noninvasive contrast imaging technique allowing quantitative visualization of living cells with subwavelength axial accuracy," Optics Letters, vol. 30, no. 5, pp. 468-470, 2005.

[38] M. K. Kim, "Principles and techniques of digital holographic microscopy," SPIE Reviews, vol. 1, no. 018005, pp. 1-50, 2010.

[39] B. Kemper, D. Carl, A. Höink, G. von Bally, I. Bredebusch, and J. Schnekenburger, "Modular digital holographic microscopy system for marker free quantitative phase contrast imaging of living cells," SPIE Proceedings, vol. 6191, p. 61910T, 2006.

[40] B. Kemper and G. von Bally, "Digital holographic microscopy for live cell applications and technical inspection," Applied Optics, vol. 47, no. 4, pp. A52-A61, 2008.

[41] M. K. Kim, Y. Hayasaki, P. Picart, and J. Rosen, "Digital holography and 3D imaging: introduction to feature issue," Applied Optics, vol. 52, no. 1, p. DH1, 2013.

[42] J. Svoboda, M. Škereň, M. Květoň, and P. Fiala, "Holographic 3D imaging-methods and applications," Journal of Physics: Conference Series, vol. 415, no. 1, article 2051, 7 pages, 2013.

[43] U. Schnars and W. Jüptner, "Direct recording of holograms by a CCD target and numerical reconstruction," Applied Optics, vol. 33, no. 2, pp. 179-181, 1994.

[44] G. Di Caprio, A. El Mallahi, P. Ferraro et al., “4D tracking of clinical seminal samples for quantitative characterization of motility parameters," Biomedical Optics Express, vol. 5, no. 3, pp. 690-700, 2014.

[45] F. Merola, L. Miccio, P. Memmolo et al., "Digital holography as a method for $3 \mathrm{D}$ imaging and estimating the biovolume of motile cells," Lab on a Chip, vol. 13, no. 23, pp. 45124516, 2013.

[46] P. Marquet, C. Depeursinge, and P. J. Magistretti, "Review of quantitative phase-digital holographic microscopy: promising novel imaging technique to resolve neuronal network activity and identify cellular biomarkers of psychiatric disorders," Neurophotonics, vol. 1, no. 2, p. 020901, 2014.

[47] Y. Hayasaki, C. Zhou, G. Popescu, and L. Onural, "Feature issue of digital holography and 3D imaging $(\mathrm{DH})$ introduction," Optics Express, vol. 22, no. 23, pp. 29117-29118, 2014.

[48] T.-W. Su, L. Xue, and A. Ozcan, "High-throughput lensfree 3D tracking of human sperms reveals rare statistics of helical trajectories," Proceedings of the National Academy of Sciences, vol. 109, no. 40, pp. 16018-16022, 2012.

[49] J. F. Jikeli, L. Alvarez, B. M. Friedrich et al., "Sperm navigation along helical paths in 3D chemoattractant landscapes," Nature Communications, vol. 6, p. 7985, 2015.

[50] S. K. Mirsky, I. Barnea, and N. T. Shaked, Label-Free Quantitative Imaging of Sperm for In-Vitro Fertilization Using Interferometric Phase Microscopy, vol. 4, no. 3, p. 1000190, 2016.

[51] J. W. Kang, N. Lue, C. R. Kong et al., "Combined confocal Raman and quantitative phase microscopy system for biomedical diagnosis," Biomedical Optics Express, vol. 2, no. 9, pp. 2484-2492, 2011.

[52] Z. Huang, G. Chen, X. Chen et al., "Rapid and label-free identification of normal spermatozoa based on image analysis and micro-Raman spectroscopy," Journal of Biophotonics, vol. 7, no. 9, pp. 671-675, 2014.

[53] S. Kosmeier, S. Zolotovskaya, A. C. De Luca et al., "Nonredundant Raman imaging using optical eigenmodes," Optica, vol. 1, no. 4, pp. 257-263, 2014.

[54] M. A. Ferrara, P. Dardano, L. De Stefano et al., "Optical properties of diatom nanostructured biosilica in Arachnoidiscus sp: micro-optics from mother nature," PLoS One, vol. 9, no. 7, p. e103750, 2014.

[55] S. Schlücker, M. D. Schaeberle, S. W. Huffman, and I. W. Levin, "Raman microspectroscopy: a comparison of point, line, and wide-field imaging methodologies," Analytical Chemistry, vol. 75, no. 16, pp. 4312-4318, 2003.

[56] B. Kemper, P. Langehanenberg, and G. Von Bally, "Digital holographic microscopy," Optik \& Photonik, vol. 2, no. 2, pp. 41-44, 2007.

[57] M. A. Ferrara, E. De Tommasi, G. Coppola, L. De Stefano, I. Rea, and P. Dardano, "Diatom valve three-dimensional representation: a new imaging method based on combined microscopies," International Journal of Molecular Sciences, vol. 17, no. 10, p. 2016, 1645.

[58] F. Dubois, M.-L. N. Requena, C. Minetti, O. Monnom, and E. Istasse, "Partial spatial coherence effects in digital holographic microscopy with a laser source," Applied Optics, vol. 43, no. 5, pp. 1131-1139, 2004.

[59] G. Rusciano, A. C. De Luca, G. Pesce, and A. Sasso, "Raman tweezers as a diagnostic tool of hemoglobin-related blood disorders," Sensors, vol. 8, no. 12, pp. 7818-7832, 2008.

[60] S. Managò, A. C. DeLuca, I. Rendina et al., "Normal-state optical features study of Nd123 and Gd1212 HTSC materials for photonics and metamaterials fabrication," IEEE Transactions on Applied Superconductivity, vol. 26, no. 3, pp. 1-4, 2016.

[61] N. Otsu, "A threshold selection method from gray-level histograms," Automatica, vol. 11, no. 285-296, pp. 23-27, 1975. 
[62] A. Soggiu, C. Piras, H. A. Hussein et al., "Unravelling the bull fertility proteome," Molecular BioSystems, vol. 9, no. 6, pp. 1188-1195, 2013.

[63] E. Canetta, M. Mazilu, A. C. De Luca et al., "Modulated Raman spectroscopy for enhanced identification of bladder tumor cells in urine samples," Journal of Biomedical Optics, vol. 16, no. 3, p. 037002, 2011.

[64] M. Nazarathy and J. Shamir, "Fourier optics described by operator algebra," JOSA, vol. 70, no. 2, pp. 150-159, 1980.

[65] G. J. Puppels, J. H. F. Olminkhof, G. M. J. Segers-Nolten, C. Otto, F. F. M. De Mul, and J. Greve, "Laser irradiation and Raman spectroscopy of single living cells and chromosomes: sample degradation occurs with $514.5 \mathrm{~nm}$ but not with 660 nm laser light," Experimental Cell Research, vol. 195, no. 2, pp. 361-367, 1991.

[66] M. Stoilovic, "Detection of semen and blood stains using polilight as a light source," Forensic Science International, vol. 51, no. 2, pp. 289-296, 1991.

[67] Y. Liu, G. J. Sonek, M. W. Berns, and B. J. Tromberg, "Physiological monitoring of optically trapped cells: assessing the effects of confinement by 1064-nm laser tweezers using microfluorometry," Biophysical Journal, vol. 71, no. 4, pp. 2158-2167, 1996.

[68] Y. Liu, D. K. Cheng, G. J. Sonek, M. W. Berns, C. F. Chapman, and B. J. Tromberg, "Evidence for localized cell heating induced by infrared optical tweezers," Biophysical Journal, vol. 68, no. 5, p. 2137, 1995.

[69] E. J. Peterman, F. Gittes, and C. F. Schmidt, "Laser-induced heating in optical traps," Biophysical Journal, vol. 84, no. 2, pp. 1308-1316, 2003.

[70] J. G. Duguid, V. A. Bloomfield, J. M. Benevides, and G. J. Thomas Jr, "DNA melting investigated by differential scanning calorimetry and Raman spectroscopy," Biophysical Journal, vol. 71, no. 6, p. 3350, 1996.

[71] J. M. Benevides, P. L. Stow, L. L. Ilag, N. L. Incardona, and G. J. Thomas Jr, "Differences in secondary structure between packaged and unpackaged single-stranded DNA of bacteriophage phi. X174 determined by Raman spectroscopy: a model for phi. X174 DNA packaging," Biochemistry (Mosc), vol. 30, no. 20, pp. 4855-4863, 1991.

[72] H. Barry and I. A. Okezie, DNA and Free Radicals, Ellis Horwood Limited, England, 1993.

[73] L. Lafleur, J. Rice, and G. J. Thomas, "Raman studies of nucleic acids. VII. Poly A. poly U and poly G. poly C," Biopolymers, vol. 11, no. 12, pp. 2423-2437, 1972.

[74] M. Mahadevan and G. Baker, "Assessment and preparation of semen for in vitro fertilization," in Clinical in Vitro Fertilization, pp. 83-97, Springer, 1984.

[75] A. Pousette, E. Akerlöf, L. Rosenborg, and B. Fredricsson, "Increase in progressive motility and improved morphology of human spermatozoa following their migration through Percoll gradients," International Journal of Andrology, vol. 9, no. 1, pp. 1-13, 1986.

[76] N. T. Tea, M. Jondet, and R. Scholler, “A "migration-gravity sedimentation" method for collecting motile spermatozoa from human semen," in In Vitro Fertilization, Embryo Transfer and Early Pregnancy, vol. 1, pp. 117-120, Springer, Netherlands, 1984.

[77] F. N. Wang, C. T. Lin, C. Y. Hong, C. H. C. Hsiung, T. P. Su, and H. D. Tsai, "Modification of the Wang tube to improve in vitro semen manipulation," Archives of Andrology, vol. 29, no. 3, pp. 267-269, 1992.

[78] E. Z. Drobnis, C. Q. ZHONG, and J. W. Overstreet, "Separation of cryopreserved human semen using Sephadex columns, washing, or Percoll gradients," Journal of Andrology, vol. 12, no. 3, pp. 201-208, 1991.

[79] M. Winterhalder and A. Zumbusch, "Beyond the borders-Biomedical applications of non-linear Raman microscopy," Advanced Drug Delivery Reviews, vol. 89, pp. 135-144, 2015.

[80] C. Zhang, D. Zhang, and J.-X. Cheng, "Coherent Raman scattering microscopy in biology and medicine," Annual Review of Biomedical Engineering, vol. 17, pp. 415-445, 2015.

[81] M. Cicerone, "Molecular imaging with CARS microspectroscopy," Current Opinion in Chemical Biology, vol. 33, pp. 179-185, 2016.

[82] C. H. Camp Jr, Y. J. Lee, J. M. Heddleston et al., "High-speed coherent Raman fingerprint imaging of biological tissues," Nature Photonics, vol. 8, no. 8, pp. 627-634, 2014.

[83] T. Ideguchi, S. Holzner, B. Bernhardt, G. Guelachvili, N. Picqué, and T. W. Hänsch, "Coherent Raman spectroimaging with laser frequency combs," Nature, vol. 502, no. 7471, pp. 355-358, 2013.

[84] Y. Jung, J. Tam, H. R. Jalian, R. R. Anderson, and C. L. Evans, "Longitudinal, 3D in vivo imaging of sebaceous glands by coherent anti-stokes Raman scattering microscopy: normal function and response to cryotherapy," The Journal of Investigative Dermatology, vol. 135, no. 1, pp. 39-44, 2015.

[85] J. W. Kang, P. T. So, R. R. Dasari, and D.-K. Lim, "High resolution live cell Raman imaging using subcellular organelle-targeting SERS-sensitive gold nanoparticles with highly narrow intrananogap," Nano Letters, vol. 15, no. 3, pp. 1766-1772, 2015.

[86] J. Kneipp and D. Drescher, "SERS in cells: from concepts to practical applications," in Frontier Surgery-Enhanced Raman Scatter Single Nanoparticles Single Cells, pp. 285-308, 2014.

[87] S. Lee, H. Chon, J. Lee et al., "Rapid and sensitive phenotypic marker detection on breast cancer cells using surfaceenhanced Raman scattering (SERS) imaging," Biosensors \& Bioelectronics, vol. 51, pp. 238-243, 2014.

[88] A. F. Palonpon, J. Ando, H. Yamakoshi et al., "Raman and SERS microscopy for molecular imaging of live cells," Nature Protocols, vol. 8, no. 4, pp. 677-692, 2013.

[89] G. Zito, G. Rusciano, G. Pesce, A. Dochshanov, and A. Sasso, "Surface-enhanced Raman imaging of cell membrane by a highly homogeneous and isotropic silver nanostructure," Nanoscale, vol. 7, no. 18, pp. 8593-8606, 2015.

[90] G. Rusciano, A. C. De Luca, G. Pesce et al., "Label-free probing of G-quadruplex formation by surface-enhanced Raman scattering," Analytical Chemistry, vol. 83, no. 17, pp. 6849-6855, 2011.

[91] D. P. Kelly, B. M. Hennelly, N. Pandey, T. J. Naughton, and W. T. Rhodes, "Resolution limits in practical digital holographic systems," Optical Engineering, vol. 48, no. 9, p. 095801, 2009. 

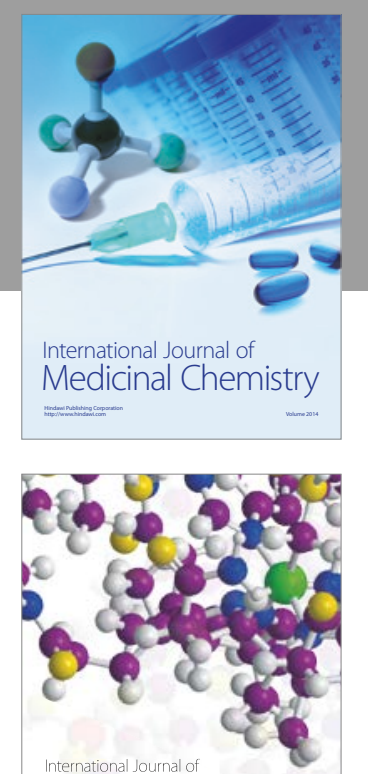

Carbohydrate Chemistry

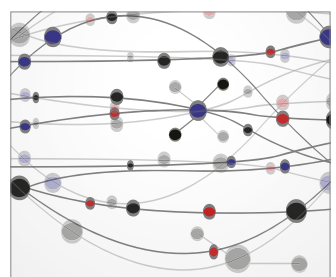

The Scientific World Journal
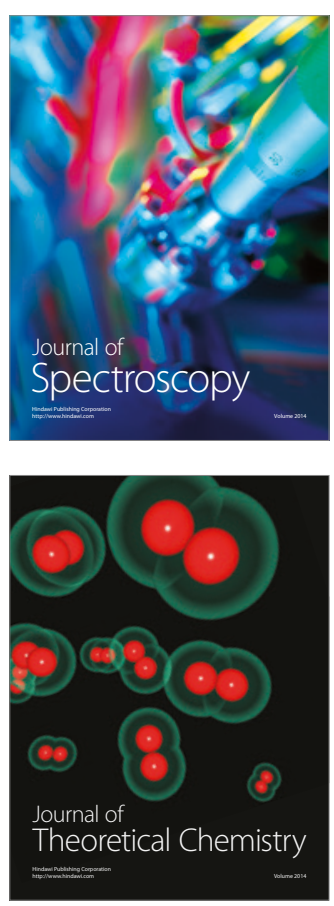
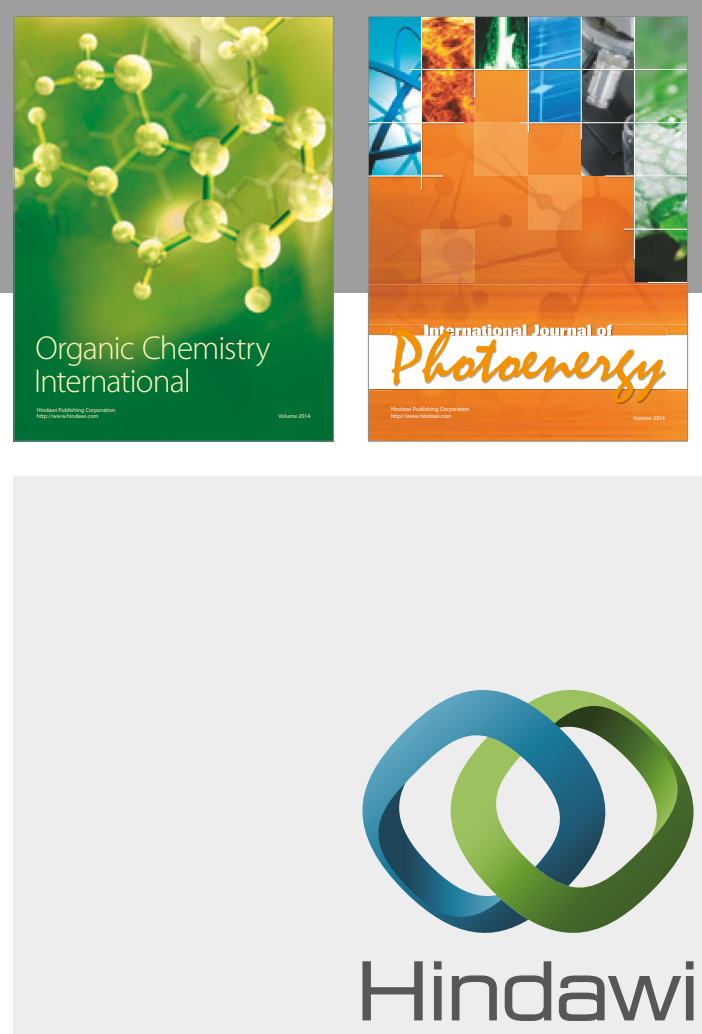

Submit your manuscripts at

https://www.hindawi.com

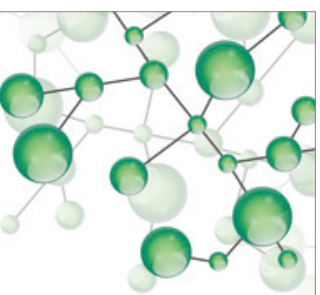

International Journal of

Inorganic Chemistry

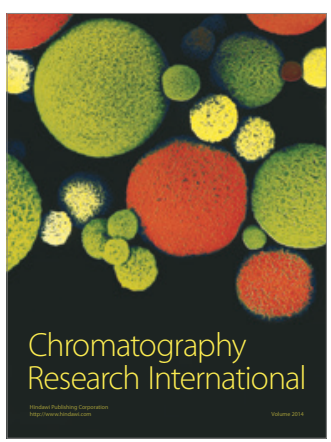

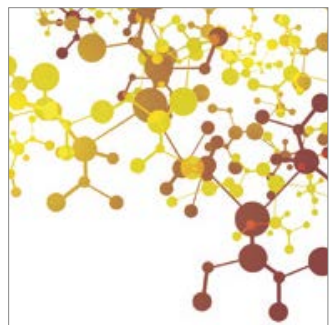

Applied Chemistry
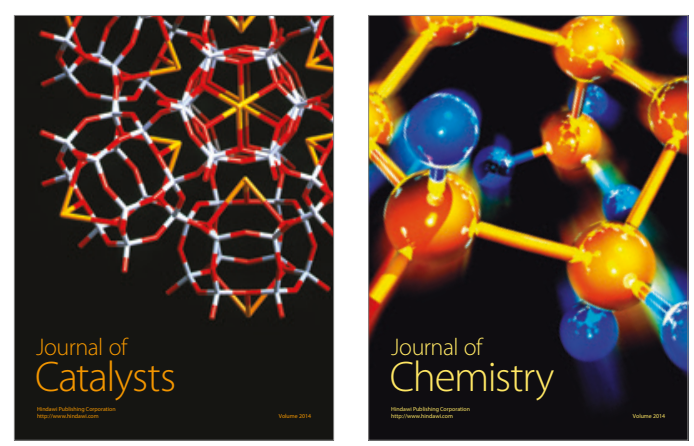
